\title{
THE USE OF. BACITRACIN FOR IDENTIFYING GROUP A HAEMOLYTIC STREPTOCOCCI
}

\author{
BY \\ W. R. MAXTED \\ From the Streptococcal Reference Laboratory, Public Health Laboratory Service, London
}

(RECEIVED FOR PUBLICATION MARCH 3, 1953)

The recognition of Lancefield's Group A as the most. commonly pathogenic members of the haemolytic streptococci has prompted several attempts at devising a rapid cultural diagnostic method such as the addition of maltose or glucose to blood agar, which alters the haemolytic activity of Group A strains (Kodama, 1937; Simmons and Wilson, 1941). This paper describes the use of an antibiotic that is more inhibitory for Group A than for other haemolytic streptococci. The investigation was begun because it was noticed that the growth of haemolytic streptococci was inhibited by a contaminating colony of an organism subsequently identified as $B$. licheniformis. Further study showed that the inhibitory agent was almost certainly identical with the antibiotic bacitracin (Johnson, Anker, and Meleney, 1945) and an extended investigation was made of the use of bacitracin for the identification of Group A streptococci.

\section{Materials and Methods}

Source of Antibiotics.-In the early work filtrates were made from cultures of $B$. licheniformis (National Collection of Type Cultures 8322) grown in nutrient broth containing $5 \%$ horse serum, or in the medium described by Hills, Belton, and Blatchley (1949). The active material is referred to as M2.

Purified samples of bacitracin and licheniformin were generously supplied by Dr. E. S. Abraham and Dr. P. D'Arcy Hart respectively.

Preparation and Testing of Antibiotics.-The early tests of M2 were carried out either with blood agar plates having an agar ditch containing approximately $4 \%$ of the filtrate, or by soaking sterile strips of filter paper in the filtrate and laying them across the inoculated zone on blood agar plates. The activity was also tested on one strain each of Groups A, B, $\mathrm{C}$, and $\mathrm{G}$ in nutrient broth containing various dilutions of the antibiotic.

For the definitive trial sterile strips of filter paper were soaked in purified bacitracin diluted 1 in 5,000 in glycine-phosphate buffer (Newton and Abraham, $1950)$; these were then dried rapidly over $\mathrm{P}_{2} \mathrm{O}_{5}$ in a desiccator, cut up into small squares, and kept in wide-mouth, screw-capped bottles in the freezing unit $\omega$ of the refrigerator (about $-2^{\circ} \mathrm{C}$.). Immediately after $N$ drying the activity of the squares was checked against susceptible, semi-resistant, and resistant Group A strains, and two members each of Groups B, C, and $G$. The average life of such squares was three weeks. More recently a solution made up with a commercial sample of bacitracin (Pfizer) containing 5 units per millilitre proved satisfactory.

Testing of Strains.-Colonies were subcultured on $\omega$ to blood agar plates and a bacitracin (or M2) paper was placed over the area of inoculation in such a position as to influence both the most heavily and less heavily inoculated zones. Plates were incubated for 24 hours. Some strains were inhibited over an area of $2 \mathrm{~cm}$. in diameter, some showed only slight inhibition, and others none at all. Those showing any inhibition from the edge of the paper were regarded as sensitive and those showing none were recorded as bacitracin (or M2) resistant. Strains growing well up to the edge of the paper but not underneath it were also regarded as resistant. Occasionally apparently haemolytic strains were non-haemolytic in the bacitracin zone but continued to grow vigorously. These also were recorded as bacitracin resistant and usually proved to be non-haemolytic when grown anaerobically.

Strains.-The initial tests of $\mathbf{M} 2$ were made on Group A strains received here for investigation as well as on representative stock strains of all the Griffith types of Group A and of all the Lancefield $N$ groups.

For the large-scale tests of bacitracin the streptococci came from three different sources. (1) All $\underset{\omega}{N}$ strains sent to the reference laboratory for typing $\widetilde{\sigma}$ during a three-month period. These had been grouped 0 and were practically all Group A. About half came from the U.S.A. and were retested at this laboratory. (2) Strains isolated from nose, throat, and other sources by the Colindale routine bacteriology department. The advantages of using these strains was $\frac{\vec{D}}{d}$ that they were from a variety of sources and districts ; in addition the examination of the plates and testing $\stackrel{\mathbb{Q}}{\Omega}$ of the strains was carried out by a number of different $\bar{O}$ workers, who, unlike those doing the tests in series 
(1) and (3) were not specialists in streptococcal work. The primary plates were incubated aerobically. (3) Strains isolated during a prolonged epidemiological survey carried out by this department in a children's home. The variety of strains was less than in (2) since single strains produced widespread infection in the home and a series of cultures was often obtained from each infected child. All primary plates were incubated anaerobically.

The strains in (1) and (2) were all tested for the Lancefield group. In (3) only those $\beta$-haemolytic cultures that were not inhibited by bacitracin were so tested as a routine. Bacitracin-sensitive $\beta$-haemolytic strains were regarded as Group A without further investigation, but were subsequently tested for type. It was thought that if the strains did not belong to Group A this would be detected by their failure to react with the type sera or by the occurrence of atypical reactions. Some members of Groups B, C, and $G$ react with' Group $A$ sera of types 2,4 , and 28 but this possibility was borne in mind. The epidemiological results suggest that errors due to this sort of cross-reaction coupled with sensitivity to bacitracin are very rare.

The enzyme grouping method (Maxted, 1948) was used throughout.

\section{Results}

The results of the preliminary tests with the M2 filtrate were as follows:

\begin{tabular}{l|l|l|r|r|r|r|r|r|r|r|r|r|r}
\hline & \multicolumn{8}{|c}{ Strains of Group } \\
\cline { 2 - 4 } & A & B & C & D & E & F & G & H & K & L & M & O \\
\hline $\begin{array}{l}\text { No. tested } \\
\text { No. inhibited }\end{array}$ & 269 & 4 & 27 & 2 & 1 & 2 & 27 & 2 & 0 & 3 & 1 & 4 \\
\hline
\end{tabular}

All the 269 Group A strains tested against M2 were inhibited compared with only eight of 73 strains of other groups, and only one of these eight strains fell into a group (G) common in the upper respiratory tract. These results indicated that further practical tests of M2 inhibition as a diagnostic method for Group A strains were justified.

Two antibiotics isolated from $B$. licheniformis, licheniformin (Callow and D'Arcy Hart, 1946; Callow, Glover, D'Arcy Hart, and Hills, 1947) and bacitracin, have previously been described. It was necessary to find out whether either of these substances was the same as M2.

The two strains of $B$. licheniformis initially used for the production of the two antibiotics, and strain 8322 , which had produced M2, were each grown in the media recommended for the production of each antibiotic, and the six filtrates tested against a variety of streptococci. It was found that M2 and bacitracin were very similar, and unlike licheniformin, in their action against streptococci.
The results of testing with bacitracin discs 3,237 strains from the three sources described are given in the table. It is clear that there was a very close relationship between bacitracin sensitivity and the serological group of haemolytic streptococci : only 22 Group A strains were found among 851 bacitracin-resistant strains and only 41 of 2,386 bacitracin-sensitive strains were found not to be Group A.

TABLE I

NUMBERS OF BACITRACIN-SENSITIVE AND RESISTANT STREPTOCOCCI FALLING INTO LANCEFIELD'S GROUPS $A, B, C, O R$ G

\begin{tabular}{|c|c|c|c|c|c|c|c|c|c|c|c|}
\hline \multirow{3}{*}{ ஜू } & \multirow{3}{*}{$\begin{array}{c}\text { Nos. } \\
\text { of } \\
\text { Strains } \\
\text { Tested }\end{array}$} & \multicolumn{5}{|c|}{ Bacitracin Sensitive } & \multicolumn{5}{|c|}{ Bacitracin Resistant } \\
\hline & & \multicolumn{4}{|c|}{$\begin{array}{l}\text { Streptococci of } \\
\text { Groups }\end{array}$} & \multirow{2}{*}{$\begin{array}{c}\text { Not } \\
\text { Group } \\
A, C, \\
\text { or } G\end{array}$} & \multicolumn{4}{|c|}{$\begin{array}{l}\text { Streptococci of } \\
\text { Groups }\end{array}$} & \multirow{2}{*}{$\begin{array}{c}\text { Not } \\
\text { Group } \\
\text { A, C, } \\
\text { or G }\end{array}$} \\
\hline & & $\mathbf{A}$ & B & $\mathrm{C}$ & $G$ & & $\mathbf{A}$ & B & C & $\bar{G}$ & \\
\hline \multirow[t]{2}{*}{$\begin{array}{l}1 \\
2 \\
3\end{array}$} & $\begin{array}{r}341 \\
1,015 \\
1,881\end{array}$ & $\begin{array}{r}328 \\
676 \\
1,341^{*}\end{array}$ & $\overline{-}$ & $\begin{array}{r}1 \\
11\end{array}$ & $\begin{array}{r}-3 \\
2\end{array}$ & $\begin{array}{r}-8 \\
14\end{array}$ & $\begin{array}{r}2 \\
6 \\
14\end{array}$ & $\overline{5}$ & $\begin{array}{r}6 \\
22 \\
251\end{array}$ & $\begin{array}{r}5 \\
16 \\
19\end{array}$ & $\begin{array}{l}\overline{283} \\
222\end{array}$ \\
\hline & 3,237 & 2,345 & 2 & 12 & 5 & 22 & 22 & 5 & 279 & 40 & 505 \\
\hline
\end{tabular}

* Most of these strains were not grouped (see text), but all were typed and reacted typically.

In some cases the apparent bacitracin resistance was due to the use of old discs and many of these strains proved to be sensitive when retested. The fact that in survey (3) a somewhat resistant strain of type 5 was rather prevalent and was encountered frequently accounts for a proportion of the 22 bacitracin-resistant Group A strains in the table. $A$ number of the strains designated " negative $A$, $\mathrm{C}$, or $\mathrm{G}$," both bacitracin sensitive and resistant, proved to be $\alpha$-haemolytic on subculture and unlikely to be confused with $\beta$-haemolytic Group A streptococci.

Bacitracin was tested under various conditions of growth to try to get even sharper distinction between Group A streptococci and members of other groups. In general this was sharper if anaerobic conditions were used throughout, but increase in the $\mathrm{CO}_{2}$ content of the atmosphere tended to increase the activity of bacitracin against all strains. The incorporation of a reducing agent such as sodium thioglycollate in the medium, although causing discoloration, did not affect the action of bacitracin on Group A strains, but rendered two Group $O$ strains, previously sensitive, resistant to the antibiotic.

For rapid identification of Group in cases of acute illness where a heavy growth of haemolytic streptococci could be expected, bacitracin paper was put on to the primary blood agar plates. Where a sufficient number of haemolytic colonies were present to make an interpretation dependable 
the results were good. There were, of course, occasions when the haemolytic colonies were so scanty as to make it impossible to assess whether the organisms were inhibited or fell outside the zone of influence of the antibiotic.

\section{Discussion}

Although there is some variation in the susceptibility of Group A streptococci to bacitracin, it is possible, by choosing the correct concentration, to use bacitracin sensitivity as a screening test in the identification of strains belonging to Lancefield's Group A. In our hands only about $1.7 \%$ of bacitracin-sensitivè and $2.8 \%$ of resistant strains would have been incorrectly diagnosed as Group A or not Group A respectively. This error is probably not much greater than may often be encountered when serological grouping is used.

As with all simple screening tests, especial care is needed in interpreting the results; bacitracin does inhibit a number of $\alpha$-haemolytic ungroupable streptococci and some micrococci, so that inhibition is only diagnostic of Group $A$ if the organism is a typical $\beta$-haemolytic streptococcus. Nevertheless the bacitracin grouping method enables the Group A strains to be recognized much sooner and with far less expenditure of work and materials than serological grouping. Its adoption can effect a great saving even if some strains have to be tested serologically because they are not inhibited or only partly inhibited by the disc. In the epidemiological survey referred to the number of strains that had to be grouped fell by approximately $80 \%$ after the adoption of the new technique.

Newton and Abraham (1950) have shown that bacitracin yields three poiypeptides, A, B, and C, each with bactericidal properties. Newton, Abra- ham, Florey, Smith, and Ross (1951) tested the bactericidal action of bacitracin $\mathrm{A}$ and $\mathrm{B}$ against $\stackrel{\vec{\rho}}{+}$ a number of organisms, including a streptococcus from each of Groups A and C, and obtained the $\frac{\vec{O}}{\bar{T}}$ same increased activity against Group $A$ as is $\frac{\bar{S}}{\overrightarrow{5}}$ shown in the present work. If more streptococci $\stackrel{\varnothing}{\circ}$ were tested it might be found that one of the polypeptides had a more selective action on Group $A$ strains than the mixture.

The therapeutic use of bacitracin may give rise $\vec{\omega}$ to bacitracin-resistant strains of Group A. This $\stackrel{\circ}{\circ}$ possibility must be borne in mind if the use of this antibiotic as a laboratory reagent is developed.

\section{Summary}

Streptosocci of Lancefield's Group A are more sensitive to bacitracin in culture media than strains of other groups, and bacitracin may therefore be $\vec{\nabla}$ used as a quick diagnostic agent for Group $A$ streptococci in the laboratory. Only $1.7 \%$ of $2,386 \frac{\mathrm{c}}{\mathrm{N}}$ bacitracin-sensitive strains proved not to be Group $A$, and only $2.5 \%$ of 851 resistant strains were $\mathscr{O}$ Group A ; in many circumstances this small proportion of incorrect diagnoses is offset by the great saving in time and material.

I wish to thank Dr. H. D. Holt and his colleagues for their co-operation in the testing of routine strains, $\frac{\varnothing}{\varnothing}$ and Dr. E. P. Abraham and Dr. P. D'Arcy Hart for $\varrho$ supplying samples of bacitracin and licheniformin.

\section{REFERENCES}

Callow, R. K., and Hart, P. D'A rcy (1946). Nature, Lond. 157, 334 . Glover, R. E., Hart, P. D'A rcy, and Hills, G. M. (1947). Brit. J. exp. Path., 28,418

Hills, G. M., Belton, F. C., and Blatchley, E. D. (1949). Ibid., 30, Johnson, B. A., Anker, H., and Meleney, F. L. (1945). Science, 102, 376.

Kodama, T. (1937). Kitasato Arch. exp. Med., 14, 29.

Maxted, W. R. (1948). Lancet, 2, 255

Newton, G. G. F., and Abraham, E. P. (1950). Biochem. J., 47, 257. О - Florey, H. W., Smith, N., and Ross, J. (1951). Brit. J. 3 Pharmacol., 6, 417.

Simmons, R. T., and Wilson, H. (1941). Med. J. Aust., 1, 74. 